\title{
Back to the Future: Academic Libraries in Transition
}

by Maxine Brodie, Macquarie University

\section{Back to the future - academic libraries in transition}

Maxine Brodie, University Librarian Macquarie University

$27^{\text {th }}$ ANZTLA Conference 12-15 July 2012

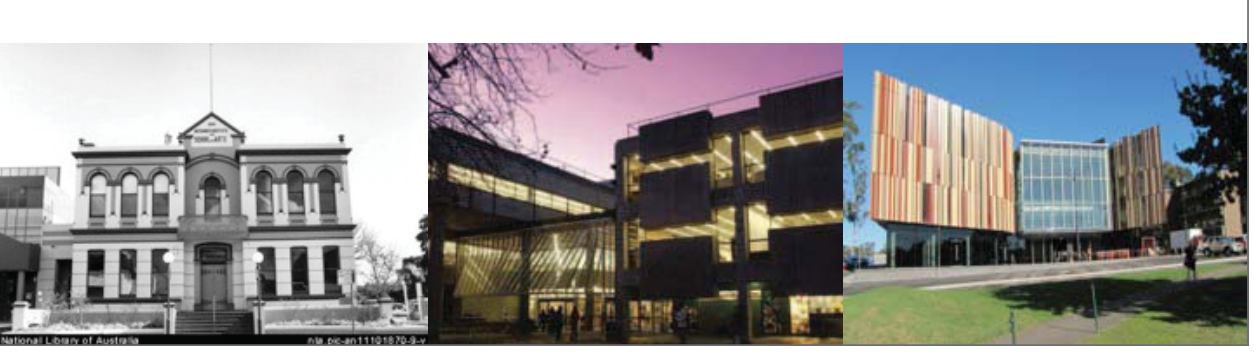




\section{What Matters?}
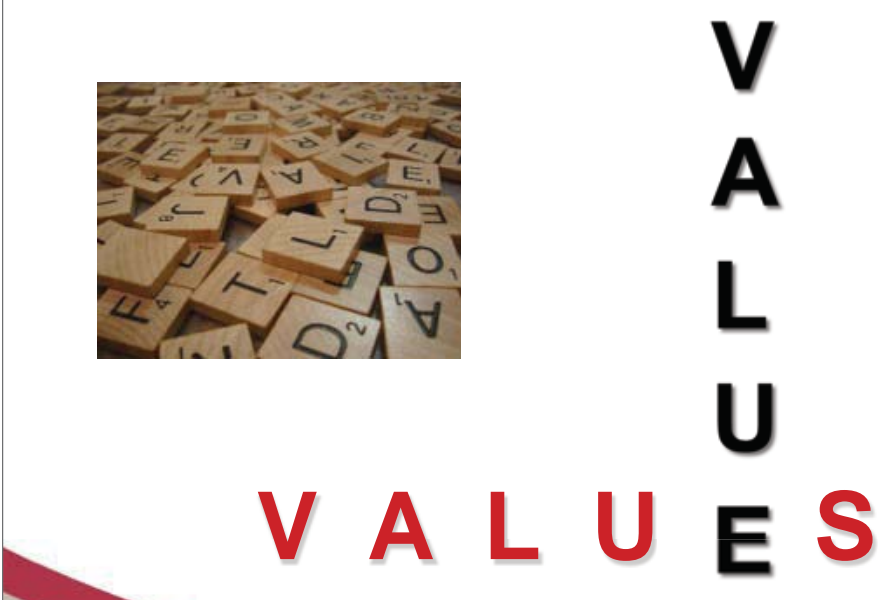

\section{Key Questions}

- What do we stand for?

(Our Values)

- What do we do that matters? (Our Value) 


\section{Professional Values}

- Promotion of the free flow of information and ideas through open access to recorded knowledge, information, and creative works.

- Connection of people to ideas.

- Commitment to literacy, information literacy and learning.

- Respect for the diversity and individuality of all people.

- Preservation of the human record.

- Excellence in professional service to our communities.

- Partnerships to advance these values.

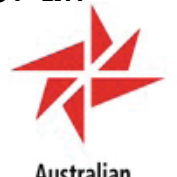

\section{Organisational Values}

\section{Does your organisation support your professional values?}

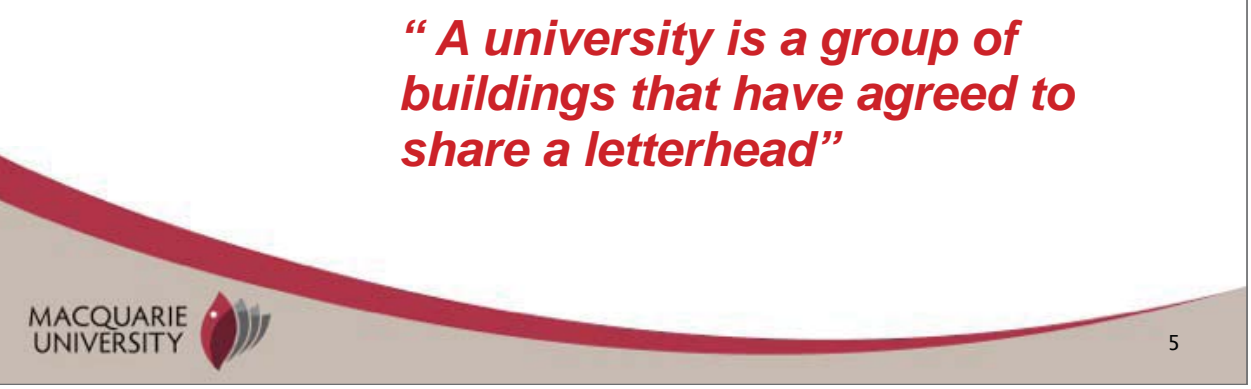




\section{What are Macquarie's Values?}
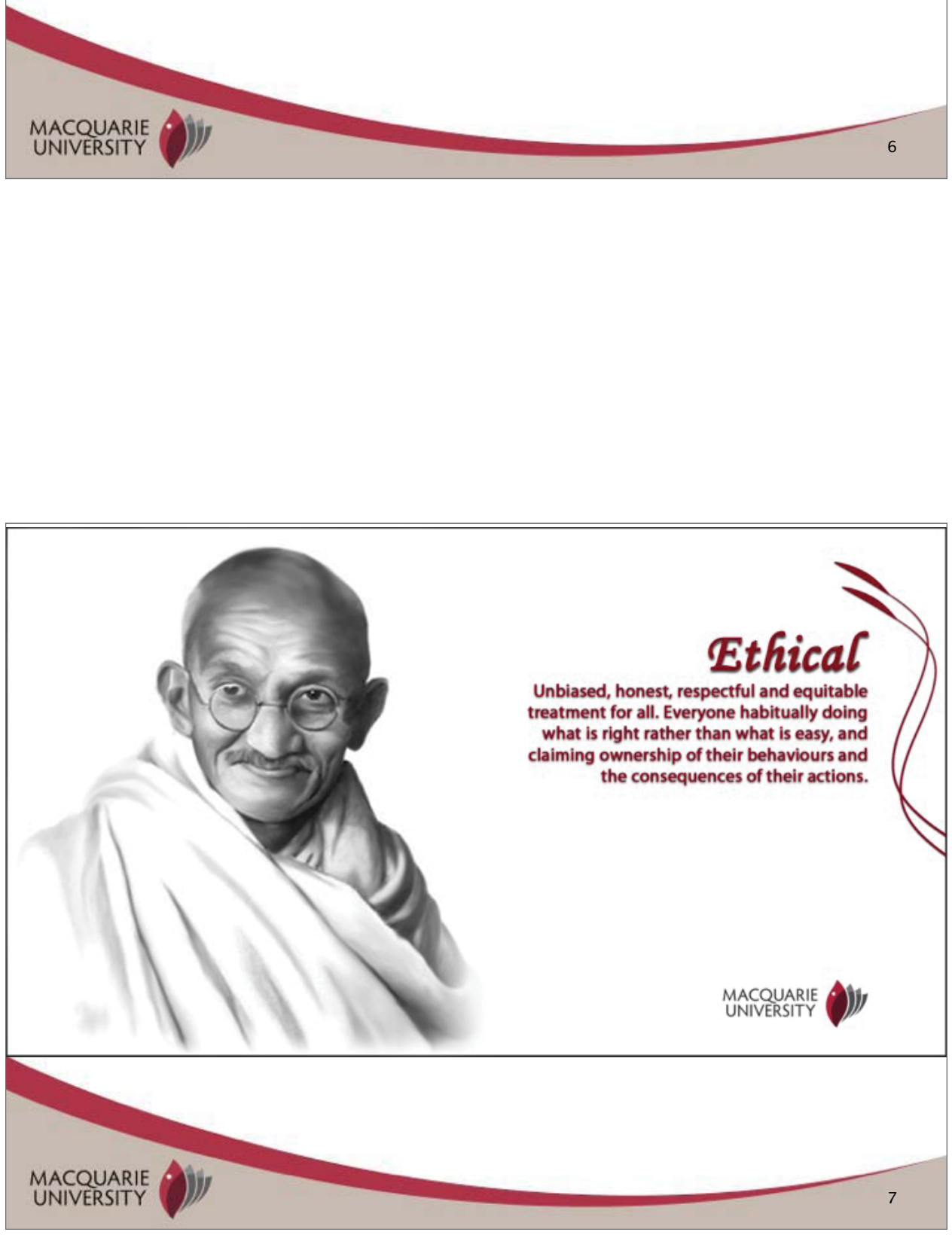


\section{Agile}

Willing and able to respond, listen and act

quickly. To be informed but not constrained by

current procedures. To be flexible, adaptable

and embrace change for positive outcomes.

MACQUARIE

UNIVERSITY
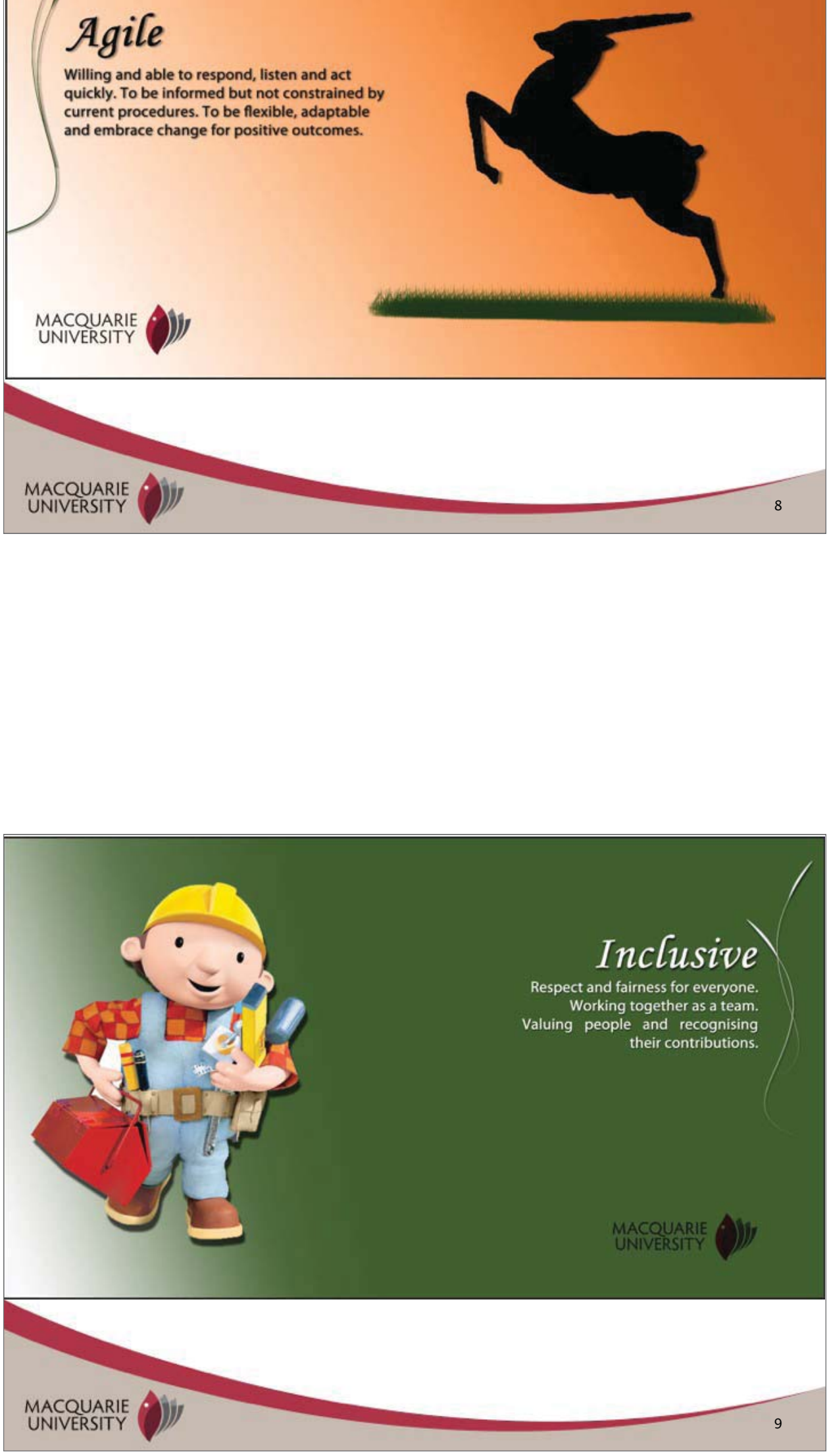

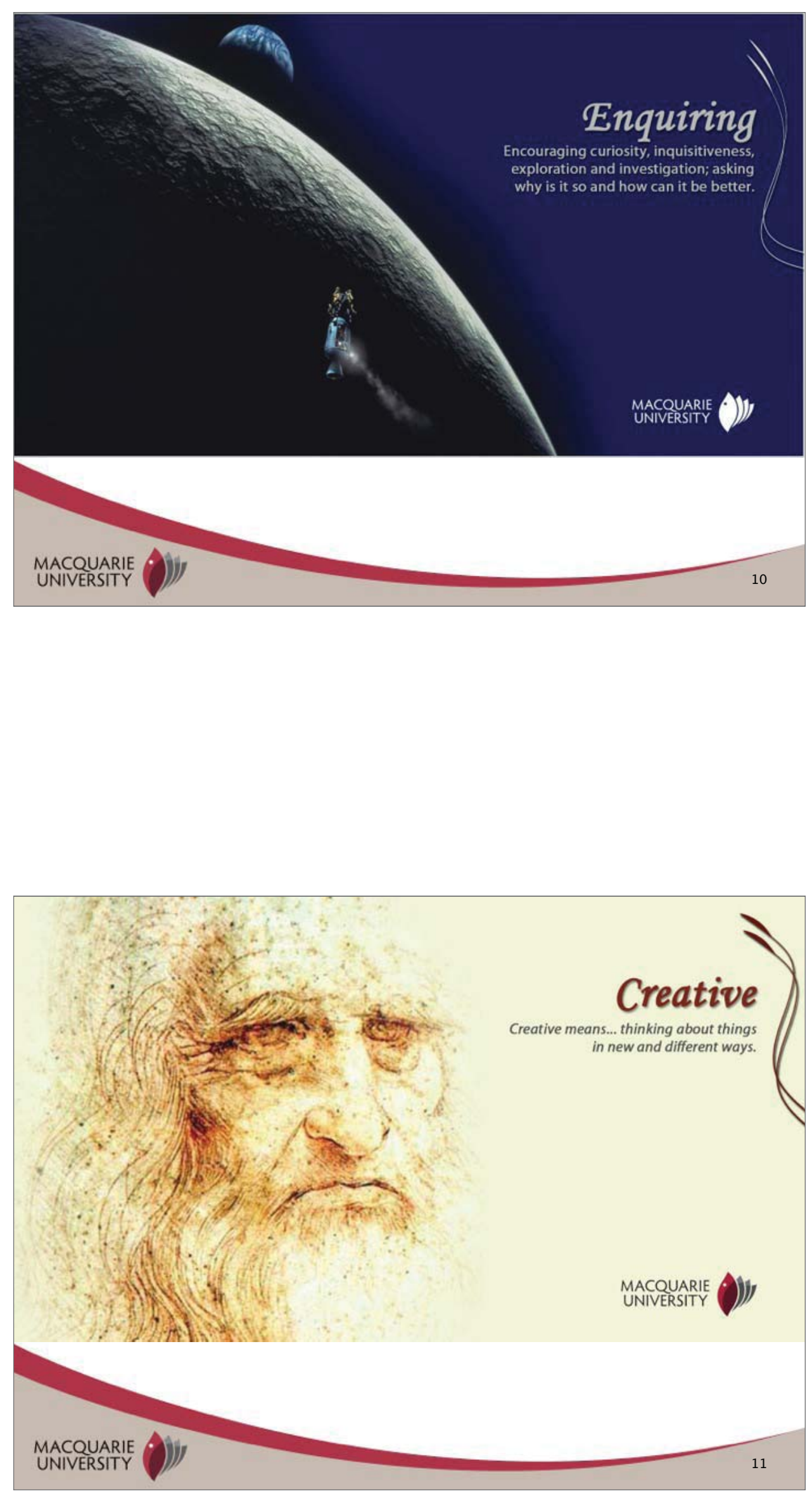


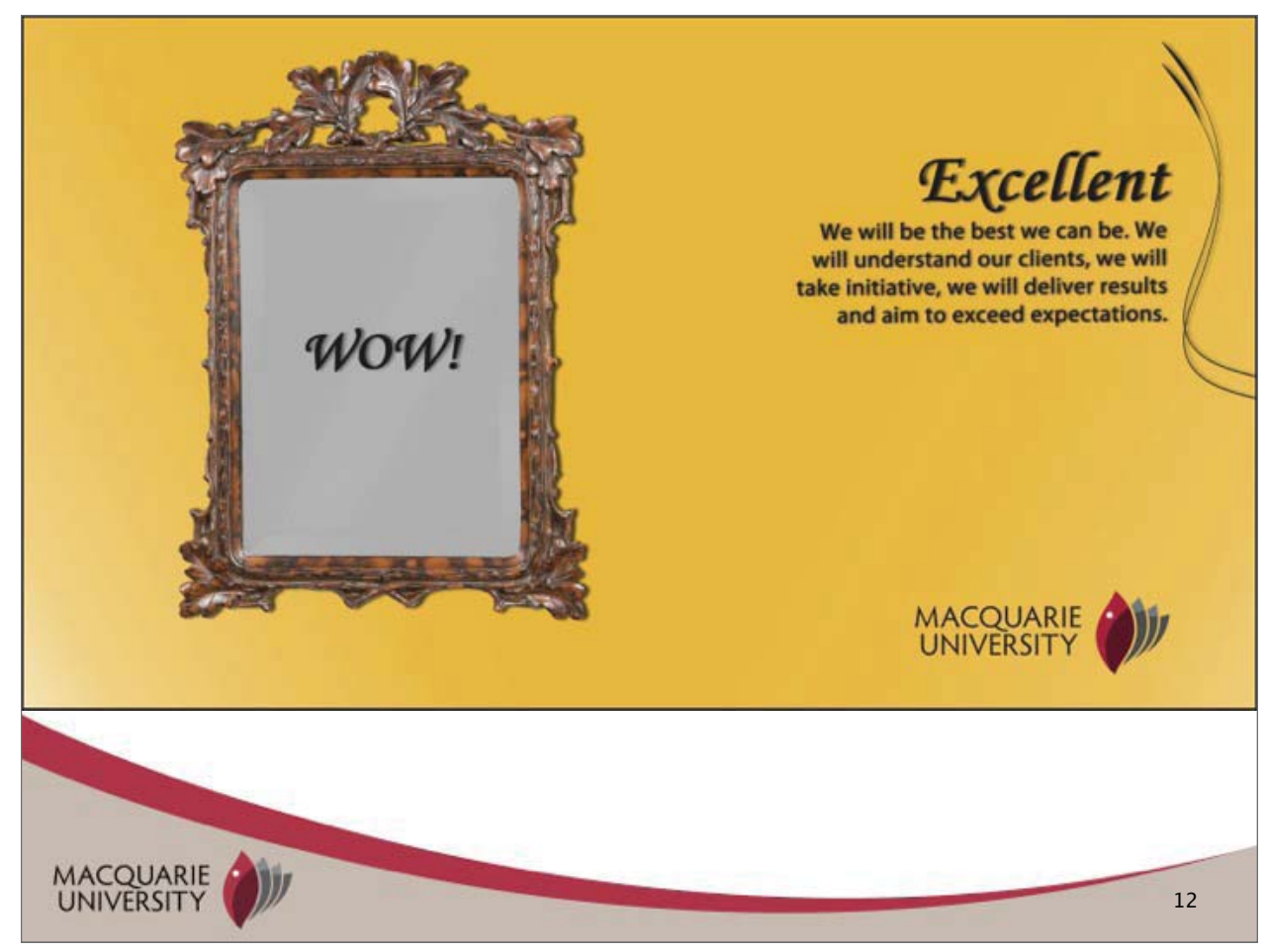

\section{So, is there a "values" fit at MQ?}

- Promotion of the free flow of information and ideas through open access to recorded knowledge, information, and creative works. (Ethical)

- Connection of people to ideas. (Enquiring)

- Commitment to literacy, information literacy and learning. (Inclusive)

- Respect for the diversity and individuality of all people. (Inclusive)

- Preservation of the human record. (?)

- Excellence in professional service to our communities. (Excellent) 


\section{So where is......}

- Creative?

- Agile?

\section{...And how important are these} for our future?

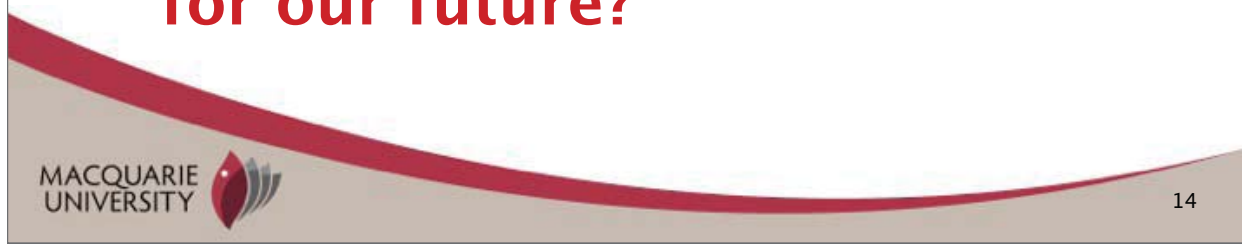

\section{Preservation of the human record}

For centuries the telling of stories has been an integral part of different cultures around the world. Mark Bahti (1988) evokes the Pueblo tradition of story-telling as follows:

"During the long winter nights when the earth, plants, animals and people were quiet and resting, awaiting the sun's return, story telling was a regular,

even nightly, event. The story took the listeners on a

journey that would bring renewal of life" (Bahti, 1988, p. 10)

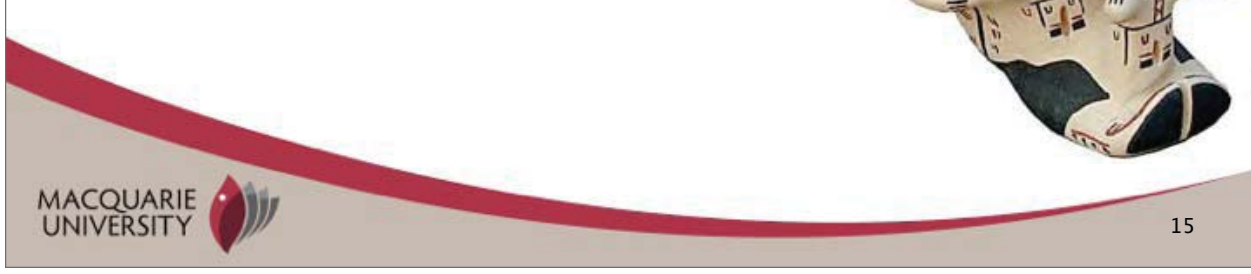




\section{Provenance}
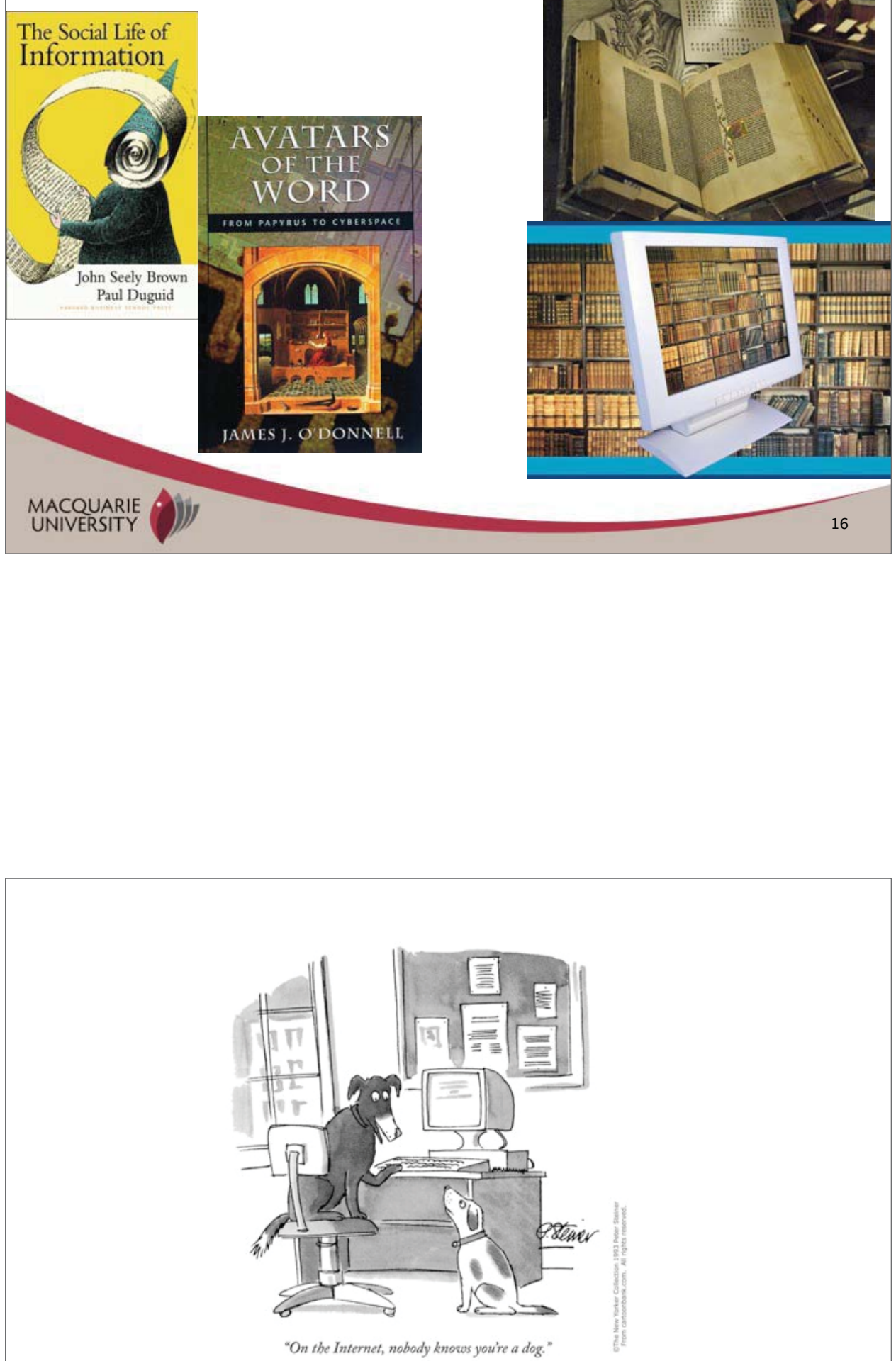

MACQUARIE 

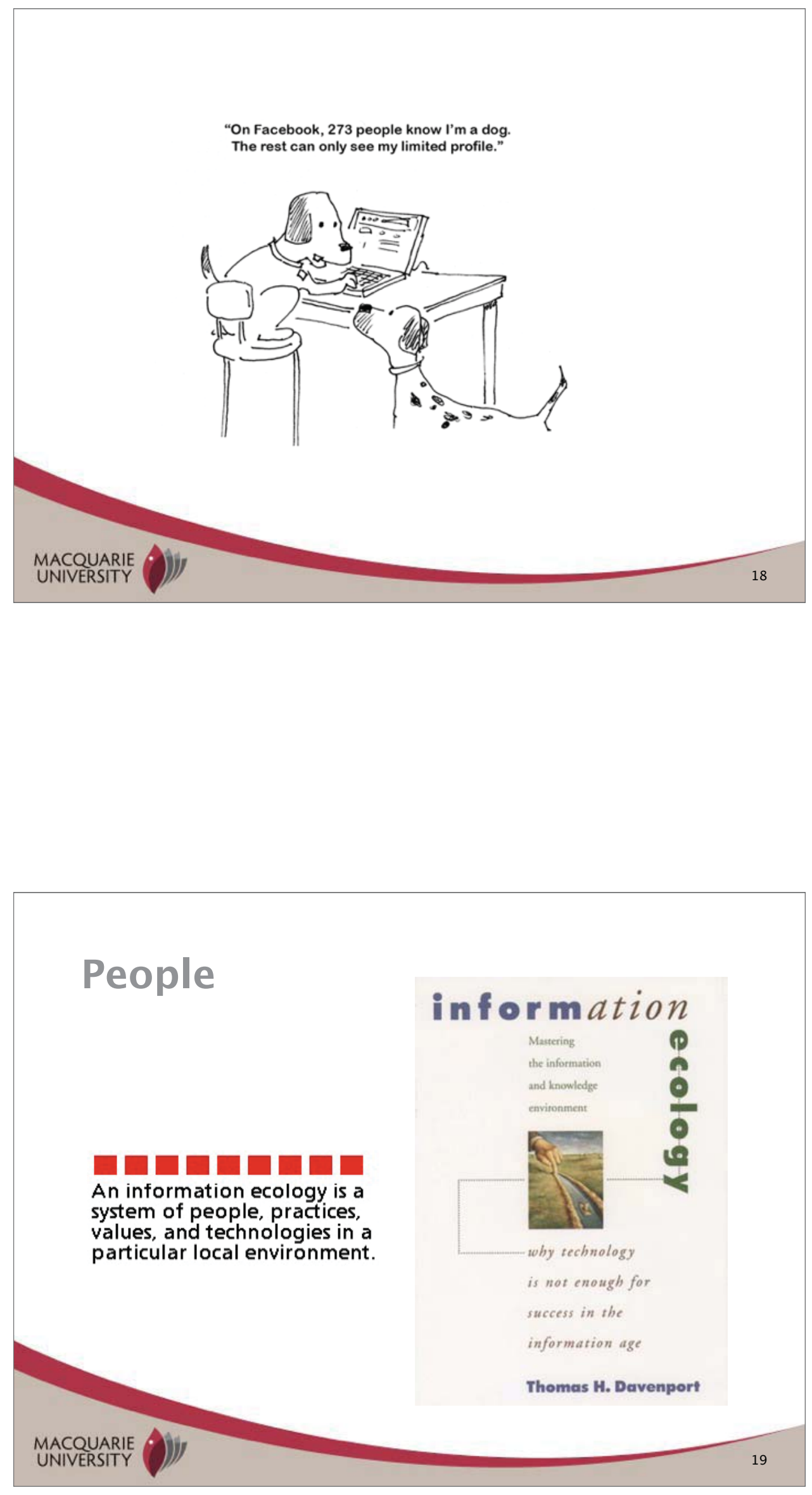


\section{A world of contradictions}

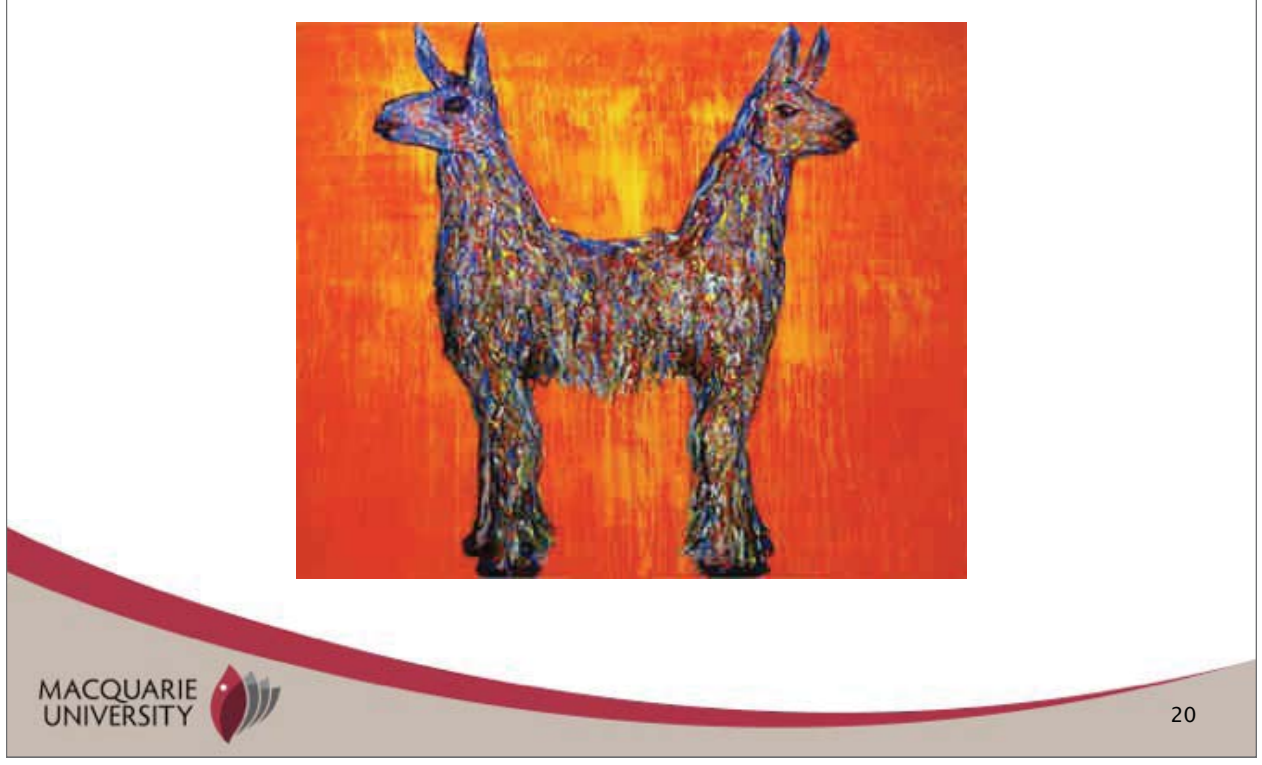

"push me, pull you"

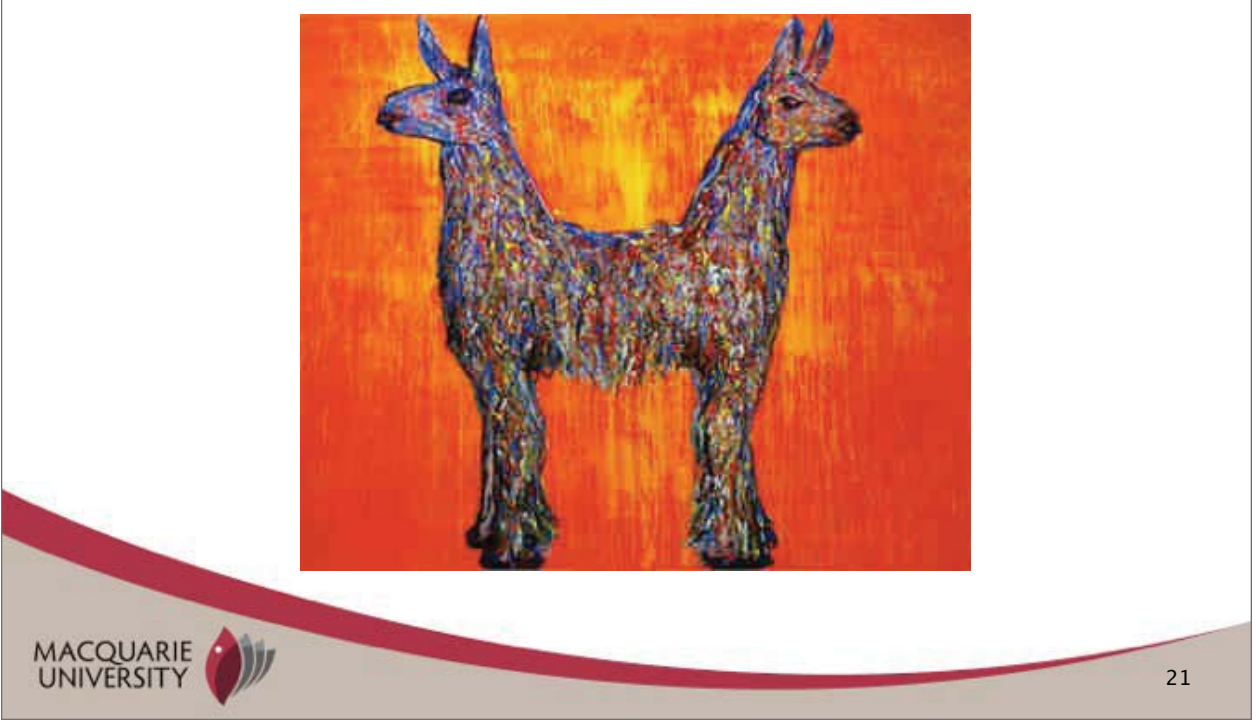




\section{Information}

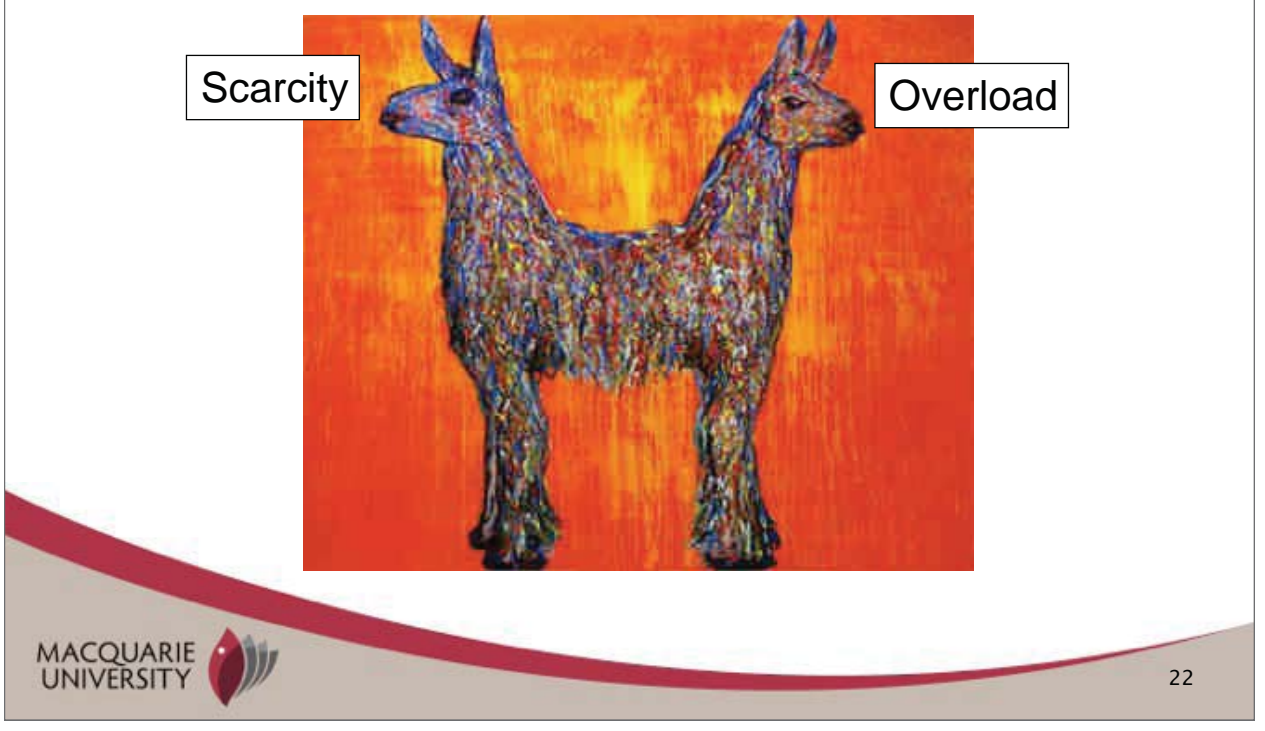

\section{Access}

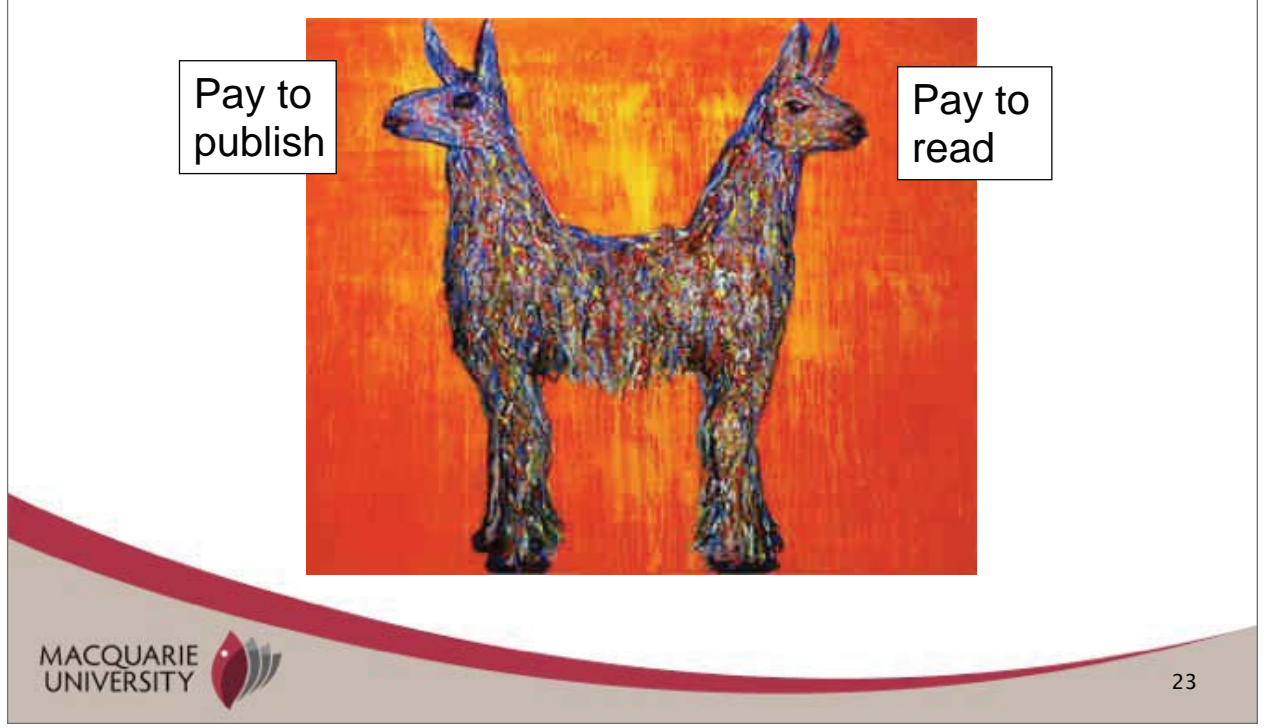




\section{Format}

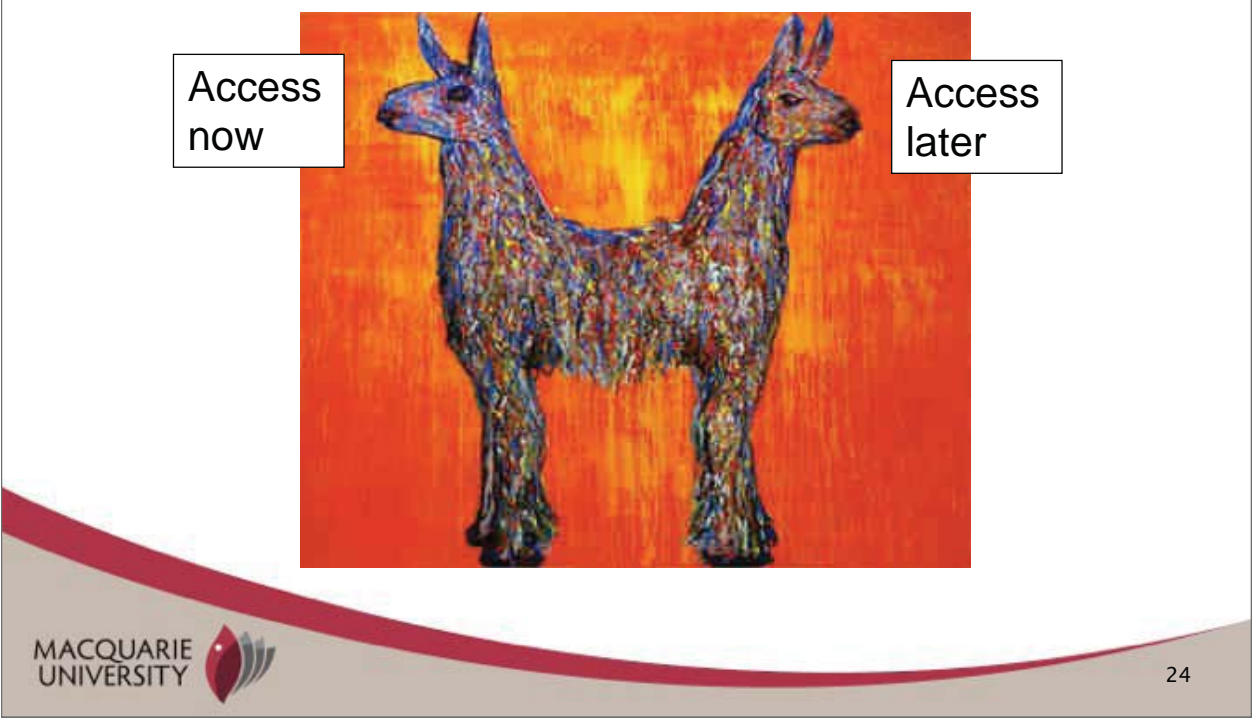

\section{Our Value}

Preservation - of knowledge, stories

Provenance - providing context, discovery

People - our skills and encouragement of interactions of people with 


\section{How our value challenges our values}

- Remember "agile" and "creative"?

- Do we see ourselves this way?

\section{Agile}

"It is better to do something imperfectly than to do nothing flawlessly"

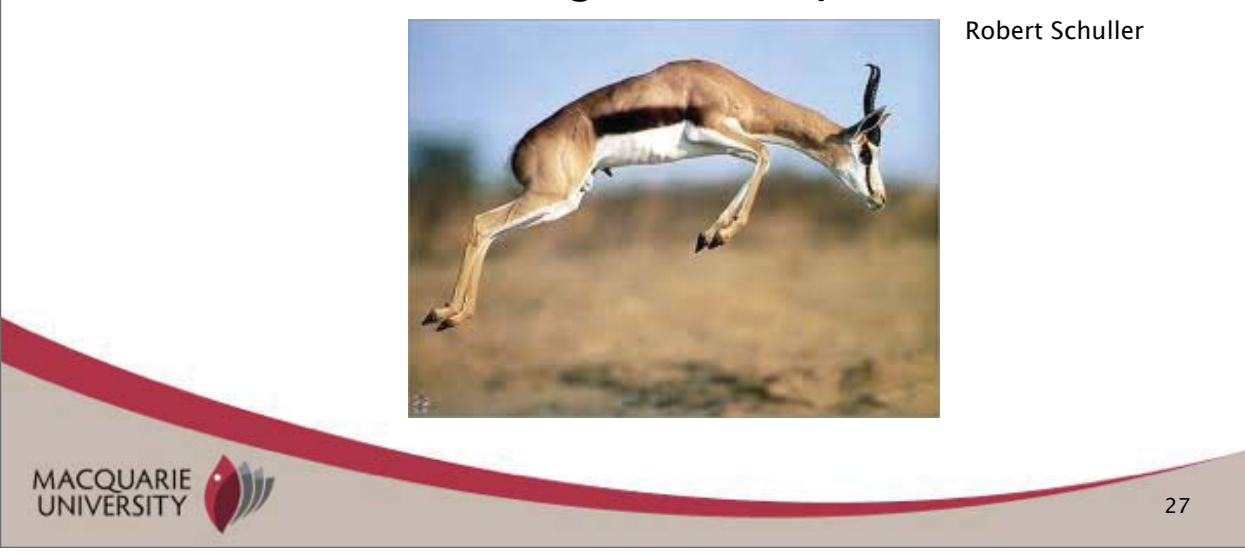




\section{Creative}

Let loose...

Work on a number of fronts at once

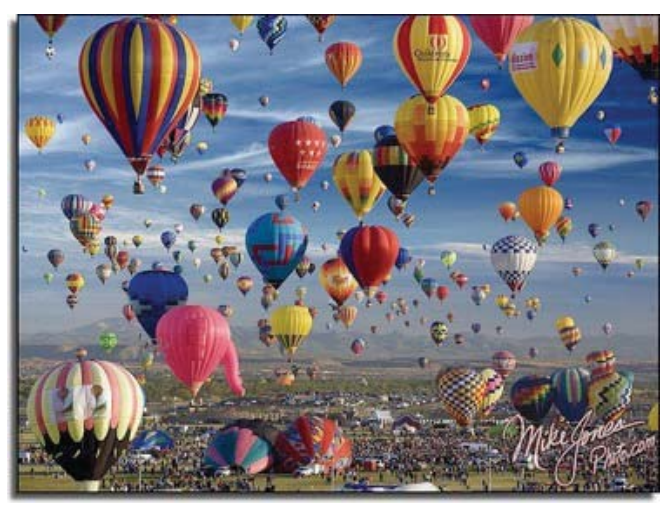

\section{So What Matters?}

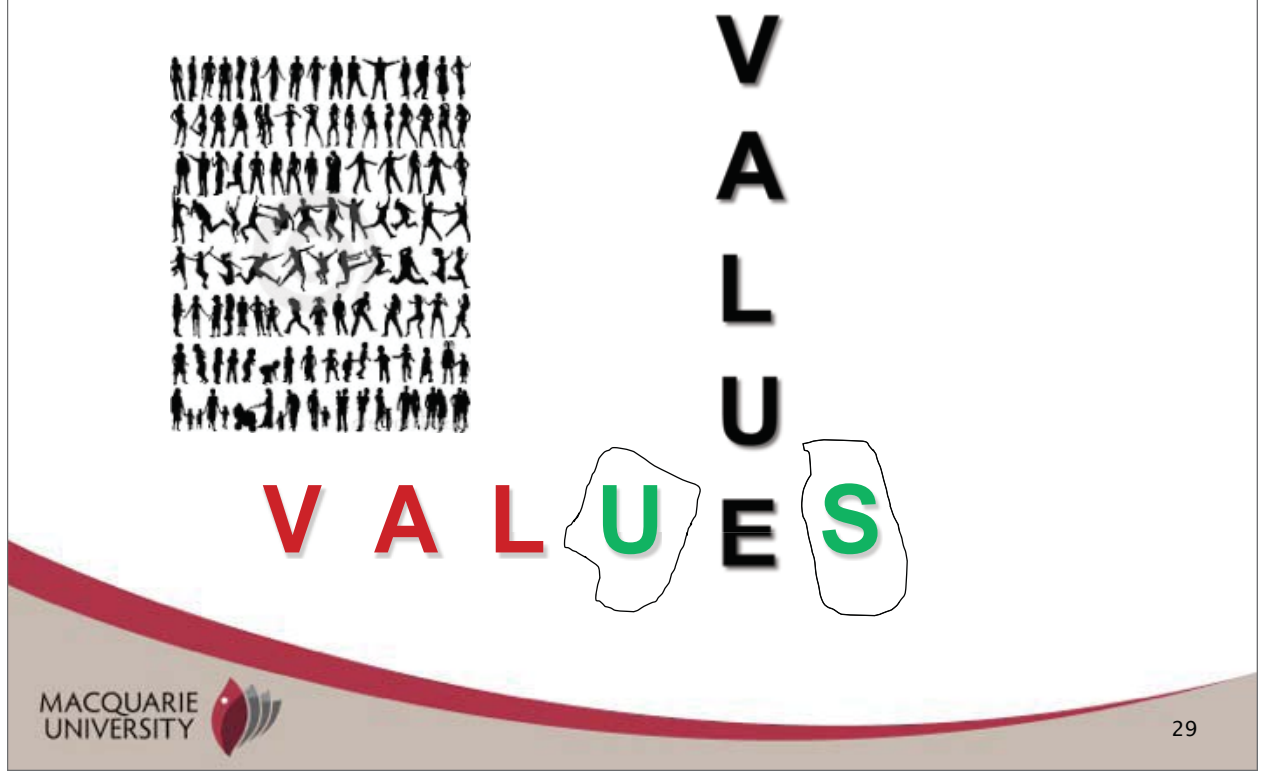




\section{Questions?}

